\title{
EQUIVARIANT COHOMOLOGY AND LOWER BOUNDS FOR CHROMATIC NUMBERS
}

\author{
IGOR KRIZ
}

\begin{abstract}
We introduce a general topological method for obtaining a lower bound of the chromatic number of an $n$-graph. We present numerical lower bounds for intersection $n$-graphs.
\end{abstract}

\section{INTRODUCTION}

An $n$-graph is a set $E$ (the edge set) whose elements are $n$-element subsets of a set $V$ (the vertex set). The chromatic number $\chi(E)$ of an $n$-graph $E$ is defined to be the minimal positive integer $t$ such that there is a mapping $\phi: V \rightarrow\{1, \ldots, t\}$ with the property that for each $M \in E,\{\phi(m) \mid m \in M\}$ has more than one element. Such a mapping is called a colouring.

The chromatic number has been an object of interest of combinatorialists for a number of years. While the upper bounds for chromatic numbers may be obtained by constructing colourings, obtaining a lower bound is in general a hard problem.

We present a method for calculating lower bounds of chromatic numbers from topological invariants. More precisely, from an $n$-graph $E$ we construct a certain partially ordered set $C$ called the resolution of $E$ (see 2.1). We show that if the homology of the classifying space $\widetilde{H}_{i}\left(B C, \mathbf{Z}_{p}\right)$ vanishes in the range $0 \leq i<(t-1)(n-1)$ for some prime $p$ which divides $n$, then $\chi(E)>t$ (see Theorem 2.2). This result was inspired by earlier work of Lovász [5] and Alon, Frankl, and Lovász [1] who used topological methods to solve conjectures of Kneser [4] and Erdös [3].

We obtain numerical lower bounds for the chromatic numbers of a particular class of $n$-graphs called intersection $n$-graphs. With a set $G$ of nonempty subsets of a set $N$ we associate an $n$-graph $[G, n]$ (called the intersection $n$ graph) and a number $w(G, n)$ (called the $n$-width). For precise definitions, see 2.3. We prove that

$$
\chi([G, n]) \geq w(G, n) /(n-1)
$$

(see Theorem 2.4).

The earlier papers $[5,1]$ show this in the case when, for some $k, G$ consists of all subsets of $N$ with $k$ elements.

Received by the editors May 7, 1990.

1980 Mathematics Subject Classification(1985 Revision). Primary 05C15, 05C65, 55N91, 55T99, $55 \mathrm{U} 99$.

Work on this paper supported in part by NSF grant DMS9002155. 
The present paper is divided into four sections. In $\S 2$ we state our main results and outline the method of their proof. Section 3 contains the calculations in Bredon cohomology which lead to the general homological lower bound. Section 4 is concerned with the case of intersection $n$-graphs.

\section{THE MAIN RESUlTS}

2.1 Definition. Let $K=(V(K), E(K))$ be an $n$-graph. Let $\mathbf{Z}_{n}$ be the cyclic group on $n$ elements with generator $a$. A resolution of $K$ is a finite partially ordered set $C$ satisfying the following axioms:

(2.1.1) The set $C_{0}$ of minimal elements of $C$ coincides with $\left\{\left(x_{1}, \ldots, x_{n}\right) \mid\right.$ $\left.\left\{x_{1}, \ldots, x_{n}\right\} \in E(K)\right\}$.

(2.1.2) The action of $\mathbf{Z}_{n}$ on $C_{0}$ given by $a \cdot\left(x_{1}, \ldots, x_{n}\right)=\left(x_{2}, \ldots, x_{n}, x_{1}\right)$ extends to a free action on $C$.

(2.1.3) Let $x \in C$ and let $x_{i}=\left(x_{i}^{1}, \ldots, x_{i}^{n}\right), i=1, \ldots, n$, be elements of $C_{0}$ such that $x_{i} \leq a^{i} x$ for all $1 \leq i \leq n$. Then $\left\{x_{1}^{1}, \ldots, x_{n}^{1}\right\} \in E(K)$.

An example of a resolution $C(K)$ of $K$ is given by letting $C(K)$ be the set of $n$-tuples $\left(M_{1}, \ldots, M_{n}\right)$ such that

(1) the $M_{i}$ are pairwise disjoint nonempty subsets of $V(K)$;

(2) if $x_{i} \in M_{i}$, then $\left\{x_{1}, \ldots, x_{n}\right\} \in E(K)$.

The partial ordering is given by

$$
\left(M_{1}, \ldots, M_{n}\right) \leq\left(N_{1}, \ldots, N_{n}\right) \text { if } M_{i} \subseteq N_{i} \text { for each } i .
$$

Recall that the classifying space $B C$ of an ordering $C$ is the realization of a simplicial set with $n$-simplices,

$$
\left[c_{0}, \ldots, c_{n}\right], \quad c_{0} \leq \cdots \leq c_{n} \in C
$$

and with the faces $\partial_{i}\left(\left[c_{0}, \ldots, c_{n}\right]\right)=\left[c_{0}, \ldots, \widehat{c_{i}}, \ldots, c_{n}\right]$ (the hat indicating omission) and degeneracies $s_{i}\left(\left[c_{0}, \ldots, c_{n}\right]\right)=\left[c_{0}, \ldots, c_{i}, c_{i}, \ldots, c_{n}\right]$. (For our purposes, we shall readily identify simplicial sets with their geometric realizations. We recall that as we factorize through both faces and degeneracies, every point in a geometrical realization is identified with a unique nondegenerate point. For more detailed information, see [6].)

2.2 Theorem. Let $K$ be an $n$-graph, let $\chi(K)=t$, and let $C$ be a resolution of $K$. Then for each prime $p$ dividing $n$ there is an $i<(n-1)(t-1)$ such that $\widetilde{H}_{i}\left(B C, \mathbf{Z}_{p}\right) \neq 0$.

It follows from the examples [1] that the bound $(n-1)(t-1)$ in the above theorem is best possible.

2.3 Definition. Let $G$ be a system of nonempty subsets of a set $N$. We define the intersection $n$-graph $[G, n]$ by putting

$$
V([G, n])=G
$$

$E([G, n])=\left\{\left\{M_{1}, \ldots, M_{n}\right\} \mid M_{i} \in G\right.$ and the $M_{i}$ are pairwise disjoint $\}$.

We also define the $n$-width of $G$ by

$w(G, n)=\min \left\{k \mid\right.$ there exist $n$ subsets $M_{i}$ of $N$ such that no subset of any

$M_{i}$ is in $G$ and the union of the $M_{i}$ has $|N|-k$ elements $\}$.

2.4 Theorem. We have $\chi([G, n]) \geq w(G, n) /(n-1)$.

In order to prove Theorem 2.2, we make the following: 
2.5 Definition. A $\mathbf{Z}_{n}$-space $S(t, n)$ is defined as follows. $S(t, n)$ is the sphere $S^{(t-1)(n-1)-1}$ viewed as the set of all $t \times n$ real matrices $\left(x_{i j}\right)_{i \in\{1, \ldots, t\}, j \in\{1, \ldots, n\}}$ such that

$$
\sum_{j=1}^{n} x_{i j}=0 \quad \text { for each } i, \quad \sum_{i=1}^{t} x_{i j}=0 \text { for each } j, \quad \sum_{i ; j} x_{i j}^{2}=1 .
$$

Recall that $a$ is a generator of $\mathbf{Z}_{n}$. The action of $\mathbf{Z}_{n}$ on $S(t, n)$ is defined by

$$
(a(x))_{i, j}=x_{i, j+1} \quad \text { for } j<n, \quad(a(x))_{i, n}=x_{i, 1} .
$$

Without difficulty, $S(t, n)$ is given a structure of a $\mathbf{Z}_{n}$-CW-complex. (This is merely a technical point. For a precise definition of a $\mathbf{Z}_{n}-\mathrm{CW}$-complex, see [2, p. I-1] or 3.1 below.)

2.6 Theorem. Let $C$ be a finite free $\mathrm{Z}_{n}-C W$-complex. Among the following statements, $(1) \Rightarrow(2) \Rightarrow(3)$.

(1) There are closed sets $C_{1}, \ldots, C_{t}$ such that $C=C_{1} \cup \cdots \cup C_{t}$ and, for each $1 \leq i \leq t, \bigcap_{g \in \mathbf{Z}_{n}} g C_{i}=\varnothing$.

(2) There is a $\mathbf{Z}_{n}$-map $\phi: C \rightarrow S(t, n)$.

(3) For each prime $p \mid n$ there is an $i<(t-1)(n-1)$ such that $\widetilde{H}_{i}\left(C, \mathbf{Z}_{p}\right) \neq 0$ (the homology taken here is nonequivariant and reduced).

We next prove the implication $(1) \Rightarrow(2)$ in Theorem 2.6. We also prove that Theorem 2.6 implies Theorem 2.2. In fact, these are the parts of our paper which are elementary. The proof of implication $(2) \Rightarrow(3)$ in Theorem 2.6 requires preliminary calculations in Bredon cohomology and will be done in $\S 3$. Theorem 2.4 is an application of our methods.

By Theorem 2.2, it suffices to construct a suitable resolution of the $n$-graph $[G, n]$ and to compute the homology of its classifying space. This will be done in $\S 4$.

2.7 Proof of (1) $\Rightarrow(2)$ in Theorem 2.6. Let $\rho$ be a metric in $C$. Let, for $x \in C$,

$$
x_{i}^{j}=\rho\left(a^{j} x, C_{i}\right), \quad x_{i}-\frac{1}{n} \sum_{j} x_{i}^{j}, \quad x^{j}=\frac{1}{t} \sum_{i} x_{i}^{j}, \quad \bar{x}=\frac{1}{n t} \sum_{i ; j} x_{i}^{j} .
$$

Put $(\tilde{\phi}(x))_{i j}=x_{i}^{j}-x_{i}-x^{j}+\bar{x}$. We compute, for a given $i, \sum_{j}(\tilde{\phi}(x))_{i j}=$ $\sum_{j}\left(x_{i}^{j}-x_{i}-x^{j}+\bar{x}\right)=n x_{i}-n x_{i}-n \bar{x}+n \bar{x}=0$, and similarly, for a given $j, \sum_{i}(\tilde{\phi}(x))_{i j}=0$. Now suppose all $(\tilde{\phi}(x))_{i j}$ are zero. Then, for each $j$, $y=x_{i}^{j}-x_{i}$ is constant in $i$ (since it equals $x^{j}-\bar{x}$ ). We have

$$
0=\sum_{i}\left(x_{i}^{j}-x_{i}\right)=t y
$$

and hence $y=0$ and $x_{i}^{j}=x_{i}$ for each $i, j$. Now $x \in C_{i}$ for some $i$. Thus, $x_{i}^{0}=0$, implying that $x_{i}^{j}=0$ for each $j$ and hence $x \in \bigcap_{g \in \mathbf{Z}_{n}} g C_{i}$, contradicting the assumption.

Thus, we may define $\phi: C \rightarrow S(t, n)$ by $\phi(x)=\tilde{\phi}(x) /\|\tilde{\phi}(x)\|$. The fact that $\phi$ is equivariant is automatic. 
2.8 Proof of Theorem 2.2. Let $\phi: V(K) \rightarrow\{1, \ldots, t\}$ be a colouring. We define, for $1 \leq i \leq t$,

$C_{i}=\bigcup\left\{\left[c_{0}, \ldots, c_{n}\right] \mid c_{0} \leq \cdots \leq c_{n} \in C \&\right.$ there exists an $\left(x_{1}, \ldots, x_{n}\right) \in C_{0}$

such that $\left(x_{1}, \ldots, x_{n}\right) \leq \dot{c}_{0}$ and $\left.\phi\left(x_{1}\right)=i\right\}$.

Obviously, $C_{i}$ are closed subsets and $B C=C_{0} \cup \cdots \cup C_{t}$. Also, the action of $\mathbf{Z}_{n}$ on $C$ extends to a free action on $B C$. Suppose that, for some $1 \leq i \leq t$,

$$
\bigcap_{g \in \mathbf{Z}_{n}} g C_{i} \neq \varnothing .
$$

Since $C_{i}$ are subcomplexes, $\bigcap_{g \in \mathbf{Z}_{n}} g C_{i}$ must contain a vertex $c$. Let, for some $x_{j}=\left(x_{j}^{1}, \ldots, x_{j}^{n}\right) \in C_{0}, x_{j} \leq a^{j} c$ and $\phi\left(x_{j}^{1}\right)=i$. Then $\phi\left(\left\{x_{1}^{1}, \ldots, x_{n}^{1}\right\}\right)=$ $\{i\}$ and, by (2.1.3), $\left\{x_{1}^{1}, \ldots, x_{n}^{1}\right\} \in E(K)$, contradicting the assumption that $\phi$ be a colouring. Thus, (2.8.1) cannot occur and we are in position to apply Theorem 2.6.

\section{EQUIVARIANT COHOMOLOGY}

The aim of this section is to prove the implication $(2) \Rightarrow(3)$ in Theorem 2.6. The proof uses some calculations in Bredon cohomology, which we do first. As a basic reference to Bredon cohomology, we recommend Bredon's book [2].

3.1 Notation. In the sequel, $n=s \cdot p_{1}^{t} \cdots p_{m}^{t_{m}}$, where the $p_{i}$ are distinct primes and $s$ is a positive integer (possibly divisible by some of the primes $p_{i}$ ). The integer $s$ is used in the induction in Theorem 3.5; it is set as 1 in the applications. In the absence of a good symbol for 'subgroup,' we shall take the liberty of using ' $\subseteq$ ' to indicate both 'subset' and 'subgroup.' The context will preclude confusion. Let $G=\mathbf{Z}_{n}$, the cyclic group on $n$ elements. Recall that a $G$ coefficient system is a set of $G$-modules $\left\{C^{(H)} \mid H \subseteq G\right\}$ and $G$-homomorphisms $r_{K}^{H}: C^{(H)} \rightarrow C^{(K)}(H \supseteq K)$ such that $C^{H}$ is $H$-fixed, and, for $H \supseteq J \supseteq K$, $r_{K}^{H}=r_{K}^{J} \circ r_{J}^{H}$. Here we are making use of the simplifications arising from the fact that $G$ is abelian (cf. [2, I.4]). $G$-coefficient systems form an abelian category $\mathfrak{C}_{G}$. The biproduct in this category will be denoted by $\oplus$.

In his book [2], Bredon defines equivariant cohomology as follows (our presentation assumes that $G$ is abelian; minor changes are needed in the general case): Let $K$ be a $G$-CW-complex, i.e., a CW-complex $K$ together with a given action of $G$ on $K$ by cellular maps such that, for each $g \in G$, $\{x \in K \mid g(x)=x\}$ is a subcomplex of $K$. For a subgroup $H \subseteq G$, we denote by $K^{H}$ the subcomplex of $K$ formed by the fixed points of $H$. Recall that, for any $\mathrm{CW}$-complex $X$, there is a chain complex $C(X)$ in the category of abelian groups where

$$
C_{n}(X)=H_{n}\left(X^{n}, X^{n-1}, \mathbf{Z}\right)
$$

and the differential $\partial: C_{n}(X) \rightarrow C_{n-1}(X)$ is the connecting homomorphism of the long exact sequence of the triple $\left(X^{n}, X^{n-1}, X^{n-2}\right)$.

Similarly, for a $G$-CW-complex $K$, we get a chain complex $\underline{C}(K)$ in the category $\mathfrak{C}_{G}$ of $G$-coefficient systems: Put

$$
\underline{C}_{n}(K)^{(H)}=C_{n}\left(K^{H}\right) \quad \text { for subgroups } H \subseteq G .
$$


The structure homomorphisms for an arbitrary $G$-coefficient system $M$, we may define a cochain complex $C_{G}(K, M)$ of abelian groups as follows:

$$
C_{G}^{n}(K)=\operatorname{Hom}\left(\underline{C}_{n}(K), M\right) .
$$

The Hom is in the category $\mathfrak{C}_{G}$. The differentials are induced from the differentials in $\underline{C}(K)$. The cohomology of $C_{G}(K, M)$ is denoted by $H_{G}^{*}(K, M)$ and called the Bredon cohomology of $K$ with coefficients in $M$.

The property of Bredon cohomology used in this paper is the Bredon spectral sequence

$$
\operatorname{Ext}^{p}\left(\underline{H}_{q}(K), M\right) \Rightarrow H_{G}^{p+q}(K, M),
$$

where Ext is in the category $\mathfrak{C}_{G}$ and $\underline{H}_{n}(K)$ is the coefficient system given by

$$
\underline{H}_{n}(K)^{(J)}=H_{n}\left(K^{J}\right) \text { for subgroups } J \subseteq G
$$

(see p. I-24 of [2]).

For a set $F$ of subgroups of $G$ (not necessarily satisfying any conditions) and for an abelian group $C$ (with trivial $G$-action) let $M(F, C)$ be the $G$ coefficient system defined by

$$
\begin{gathered}
M(F, C)^{(H)}= \begin{cases}C & \text { if } H \in F, \\
0 & \text { otherwise, }\end{cases} \\
r_{K}^{H}= \begin{cases}\operatorname{Id}_{C} & \text { if } H \supseteq J \supseteq K \text { implies } J \in F, \\
0 & \text { otherwise. }\end{cases}
\end{gathered}
$$

We also put $M(F)=M(F, \mathbf{Z})$. Note that an inclusion $F \subseteq F^{\prime}$ of sets of subgroups induces a mapping $M(F) \rightarrow M\left(F^{\prime}\right)$ of coefficient systems if for every pair of subgroups $H \supseteq J$ of $G$ such that $J \in F^{\prime}$ and $H \in F$ we have $J \in F$. For a subgroup $A \subseteq G$, let $\bar{A}$ be the system of all subgroups of $A$. Similarly to the above, we may define coefficient systems $M(F, C)$ if $F \subseteq \bar{A}$ and $C$ is an $A$-fixed $G$-module. Now put

$$
A_{i}=\mathbf{Z}_{n / s p_{i}}, \quad M=M\left(\bigcap_{i=1}^{m} \bar{A}_{i}, \mathbf{Z}_{p_{k}}\right),
$$

where $k$ is an arbitrary number $(1 \leq k \leq m)$ fixed throughout this section.

3.2 Lemma. Let $\eta: \overline{0} \rightarrow \bigcap_{i=1}^{m} \bar{A}_{i}$ be the inclusion, then

$$
\eta^{*}: \operatorname{Ext}^{*}\left(M\left(\bigcap_{i=1}^{m} \bar{A}_{i}\right), M\right) \rightarrow \operatorname{Ext}^{*}(M(\overline{0}), M)
$$

is iso. The right-hand side is $\mathbf{Z}_{p_{k}}$ in each nonnegative dimension.

Proof. The last statement is obvious [2, p. I-25, Example 1]. To see that $\eta^{*}$ is iso, observe that for any subgroup $A \subseteq G, M(\bar{A})$ has a projective resolution of the form $\left\{M\left(\bar{A}, C_{i}\right)\right\}$, where $C_{i}$ is a free $G / A$-resolution of $\mathbf{Z}$ (cf. [2, p. I-23]). Thus, we may view $\eta^{*}$ as the morphism $\zeta^{*}: H^{*}\left(\mathbf{Z}_{q s}, \mathbf{Z}_{p_{k}}\right) \rightarrow H^{*}\left(\mathbf{Z}_{n}, \mathbf{Z}_{p_{k}}\right)$, where $q=\prod_{i=1}^{m} p_{i}$ and $\zeta: \mathbf{Z}_{n} \rightarrow \mathbf{Z}_{q s}$ is given by $\zeta(1)=1$.

Now let $F$ be a set of subgroups of $G$. We call a $G$-coefficient system $C$ based at $F$ if for each subgroup $K \subseteq G$ and each $x \in C^{K}$ there is an $H \supseteq K$ and a $y \in C^{H}$ such that $H \in F$ and $x=r_{K}^{H}(y)$. 
3.3 Lemma. Let $B_{i} \subseteq G, i \in S$. Then $M\left(\bigcup_{i \in S} \bar{B}_{i}\right)$ has a projective resolution each term of which is based at the set $\left\{\bigcap_{i \in T} B_{i} \mid T \subseteq S \& T \neq \varnothing\right\}$.

Proof. The proof is by an easy induction of $|S|$ using the exact sequence

$$
0 \rightarrow M\left(\bar{B}_{t} \cap \bigcup_{i \in S \backslash\{t\}} \bar{B}_{i}\right) \rightarrow M\left(\bigcup_{i \in S \backslash\{t\}} \bar{B}_{i}\right) \oplus M\left(\bar{B}_{t}\right) \rightarrow M\left(\bigcup_{i \in S} \bar{B}_{i}\right) \rightarrow 0
$$

and the algebraic mapping cone construction.

\subsection{Lemma.}

$$
\begin{gathered}
\operatorname{Ext}^{*}\left(M\left(\bar{A}_{1}\right), M\right)=0 \quad \text { if } m>1 . \\
\operatorname{Ext}^{*}\left(M\left(\bigcup_{i=2}^{m} \bar{A}_{i}\right), M\right)=0 .
\end{gathered}
$$

Proof. By Lemma 3.3, the relevant projective resolutions are based at subgroups at which $M$ vanishes.

3.5 Theorem. Let $l: \overline{0} \rightarrow \bigcup_{i=1}^{m} \bar{A}_{i}$ be the inclusion. Then

$$
\imath^{*}: \operatorname{Ext}^{*}\left(M\left(\bigcup_{i=1}^{m} \bar{A}_{i}\right), M\right) \rightarrow \operatorname{Ext}^{*}(M(\overline{0}), M)
$$

is iso in dimensions $\geq m-1$.

Proof. We denote $\operatorname{Ext}^{*}(N)=\operatorname{Ext}^{*}(N, M)$. In view of Lemma 3.2, it suffices to consider the inclusion $\kappa: \bigcap_{i=1}^{m} \bar{A}_{i} \rightarrow \bigcup_{i=1}^{m} \bar{A}_{i}$ and prove that

$$
\kappa^{*} \text { is iso in dimensions } \geq m-1 \text {. }
$$

This shall be proved by induction on $m$. For $m=1$ the statement is trivial. Let $m>i$ and let the statement be true with $m$ replaced by $m-1$. Put $s^{\prime}=s p_{1}$. By the induction hypothesis applied to $A_{2}, \ldots, A_{m} \subseteq \mathbf{Z}_{n}, s$ replaced by $s^{\prime}$, the inclusion $\lambda: \bigcap_{i=1}^{m} \bar{A}_{i} \rightarrow \bar{A}_{1} \cap \bigcup_{i=2}^{m} \bar{A}_{i}$ satisfies

$$
\lambda^{*} \text { is iso in dimensions } \geq m-2 \text {. }
$$

We now consider the following diagram, which arises from Mayer-Vietoris exact sequences:

$$
\begin{aligned}
& \operatorname{Ext}^{t-1}\left(M\left(\bigcup_{i>1} \bar{A}_{i}\right) \oplus M\left(\bar{A}_{1}\right)\right) \\
& \beta \\
& \operatorname{Ext}^{t-1}\left(M\left(\bigcup_{i>1} \bar{A}_{i} \cap \bar{A}_{1}\right)\right) \\
& \delta\rfloor \\
& \operatorname{Ext}^{t}\left(M\left(\bigcup_{i>1} \bar{A}_{i} \backslash \bar{A}_{1}\right) \oplus M\left(\bar{A}_{1} \backslash \bigcup_{i>1} \bar{A}_{i}\right)\right) \stackrel{\gamma}{\longrightarrow} \operatorname{Ext}^{t}\left(M\left(\bigcup_{i>1} \bar{A}_{i} \cup \bar{A}_{1}\right)\right) \\
& \stackrel{\alpha}{\longrightarrow} \operatorname{Ext}^{t}\left(M\left(\bigcup_{i>1} \bar{A}_{i} \cap \bar{A}_{1}\right)\right) \text {. }
\end{aligned}
$$


By Lemma 3.4, $\beta=0$ (the source is 0$)$ and $\lambda=0(\gamma$ factors through $\left.\operatorname{Ext}^{t}\left(M\left(\bigcup_{i>1} \bar{A}_{i}\right) \oplus M\left(\bar{A}_{1}\right)\right)\right)$. Thus, $\delta$ and $\alpha$ are mono. In view of the fact that the source of $\delta$ and the target of $\alpha$ are both $\mathbf{Z}_{p_{k}}$ for $t-1 \geq m-2$ (by (3.5.2) and Lemma 3.2), we conclude that in that range $\alpha$ is iso. Together with (3.5.2) this gives the result.

We are now ready to finish the proof Theorem 2.6.

3.6 Proof of the implication (2) $\Rightarrow(3)$ in Theorem 2.6. If $C$ is not connected, we are done. Thus, assume that $C$ is connected. Recall that $C$ is free. We now adopt the notation of $3.1(s=1)$. We shall consider Bredon cohomology with coefficients in $M$ (as in 3.1). The mapping $\phi: C \rightarrow S(t, n)$ induces a mapping in Bredon cohomology

$$
\phi^{*}: H_{G}^{*}(S(t, n), M) \rightarrow H_{G}^{*}(C, M)
$$

and hence a mapping of Bredon spectral sequences

$$
\phi_{r}^{*}: E_{r}^{p, q}(S(t, n)) \rightarrow E_{r}^{p, q}(C),
$$

where

$$
E_{2}^{p, q}(X)=\operatorname{Ext}^{p}\left(\underline{H}_{q}(X), M\right) .
$$

(Here, following Bredon [2], $\underline{H}_{q}(X)^{(H)}=H_{q}\left(X^{H}\right)$.) We now observe that

$$
\underline{H}_{0}(C)=M(\overline{0}),
$$

and, unless $t=2$ and $2 \mid n$,

$$
\underline{H}_{0}(S(t, n))=M\left(\bigcup_{i=1}^{m} \bar{A}_{i}\right) .
$$

Assume (3.6.4) for now; the case when $t=2$ and $2 \mid n$ will be discussed at the end of the proof. By Theorem 3.5, $\phi_{2}^{p, q}$ is iso for $p=(t-1)(n-1) \geq$ $m-1$ and $q=0$. Now $E_{2}^{(t-1, n-1), 0}(S(t, n)$ ) (which is nonzero by Lemma 3.2) is bound to die in the spectral sequence (for we have

$$
\operatorname{dim} S(t, n)=(t-1)(n-1)-1)
$$

and hence $E_{2}^{(t-1, n-1), 0}(C)$ is bound to die, too (by the naturality of differentials). However, the latter can only get hit by a differential starting in the range $q<(t-1)(n-1)$. This gives the desired result by (3.6.2). The above procedure has a slight defect in the case $t=2 \& 2 \mid n$. Then, for $k=2$ $S(t, n)^{\mathbf{Z}_{n / k}}=S(t, k)=S(2,2)=S^{0}$ so that $\underline{H}_{0}(S(2,2))^{\left(\mathbf{Z}_{n / k}\right)}$ is $\mathbf{Z} \oplus \mathbf{Z}$ rather than $\mathbf{Z}$. Instead of playing any more games with the coefficients, we choose to resolve this difficulty geometrically. Let $\bar{S}(t, n)$ be the pushout

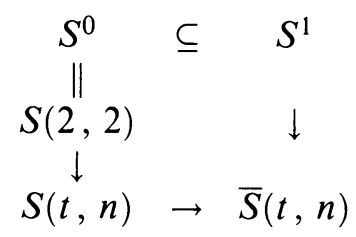

(the top line inclusion being viewed as $\{(1,0),(-1,0)\} \subseteq\left\{x \in \mathbf{R}^{2} \mid\|x\|=1\right\}$ ) and extend the action of $\mathbf{Z}_{n}$ to $\bar{S}(t, n)$ by putting $a \cdot x=-x$ for $x \in S^{1}$ 
(recall that $a$ is the generator of $\mathbf{Z}_{n}$ ). Now $\phi$ may certainly be viewed as a $\mathbf{Z}_{n}$-mapping to $\bar{S}(t, n)$ and $\underline{H}_{0}(\bar{S}(t, n))=M\left(\bigcup_{i=1}^{m} \bar{A}_{i}\right)$.

\section{Calculations for intersection $n$-GRaPhS}

To prove Theorem 2.4 , we define a resolution $Z(G, n)$ of $[G, n]$ by letting $Z(G, n)$ be the set of $n$-tuples $\left(M_{1}, \ldots, M_{n}\right)$ such that

(1) the $M_{i}$ are pairwise disjoint subsets of $N$; and

(2) each $M_{i}$ contains a subset which belongs to $G$.

The partial ordering is given by

$$
\left(M_{1}, \ldots, M_{n}\right) \leq\left(N_{1}, \ldots, N_{n}\right) \text { if } M_{i} \subseteq N_{i} \text { for each } i .
$$

Observe that this is not the resolution $C([G, n])$ as constructed in 2.1. Theorem 2.4 now follows from the following result:

4.1 Theorem. For $0 \leq i<w(G, n)-n$ we have $\widetilde{H}_{i}(B Z(G, n), \mathbf{Z})=0$.

Proof. We have a natural embedding $Z(G, n) \subseteq \prod_{i=1}^{n} Z(G, 1)$ and hence

$$
B Z(G, n) \subseteq \prod_{i=1}^{n} B Z(G, 1) .
$$

This formula will be used to calculate the homology. We shall proceed by induction on $n$. For $n=1$ the space $B Z(G, 1)$ is actually contractible since $Z(G, 1)$ has a largest element $N$. For a simplex $s=\left[M_{1} \subseteq \cdots \subseteq M_{i}\right] \in$ $B Z(G, 1)$, put $\bar{s}=M_{i}$ and $s^{\prime}=\left\{M_{1}, \ldots, M_{i-1}\right\}$. In the sequel, a simplex means a nondegenerate simplex.

Now fix $n$ and let the statement of Theorem 4.1 hold with $n$ replaced by $n-1$. Now by the definition of $Z(G, n)$ together with $(4.1 .1), H_{*}(B Z(G, n))$ is the homology of the complex $C$ where $C_{*}$ is the free abelian group with basis

$$
\left\{s_{1} \otimes \cdots \otimes s_{n} \mid i \neq j \Rightarrow \bar{s}_{i} \cap \bar{s}_{j}=\varnothing\right\}
$$

and the boundary operator is the obvious one. We filter $C$ by putting

$$
F_{j} C=F\left\{s_{1} \otimes \cdots \otimes s_{n} \mid \operatorname{dim} s_{1} \leq j\right\}
$$

( $F X$ denotes the free abelian group on the set $X$ ). The resulting spectral sequence collapses to the $E^{2}$-term. This is immediate because the boundary operator never decreases filtration degree by more than one. To describe the $E^{1}$-term, let $G_{\bar{s}}=G_{s}=G \cap 2^{N \backslash \bar{s}}$ for a simplex $s . G_{\bar{s}}$ will be viewed as a set system on $N \backslash \bar{s}$. Putting

$$
e(s)=\max \left\{|M| \mid M \subseteq \bar{s} \& G \cap 2^{M}=\varnothing\right\}
$$

we observe that

$$
w\left(G_{s}, n-1\right) \geq w(G, n)-|\bar{s}|+e(s) .
$$

Now for $t \subseteq s$ we have $G_{s} \subseteq G_{t}$, hence $Z\left(G_{s}, n-1\right) \subseteq Z\left(G_{t}, n-1\right)$ and hence there is a homomorphism

$$
r_{s t}: H_{q}\left(B Z\left(G_{s}, n-1\right)\right) \rightarrow H_{q}\left(B Z\left(G_{t}, n-1\right)\right) .
$$


Now we have

$$
E_{p, q}^{1}=\sum\left\{s \otimes \alpha \mid \operatorname{dim} s=p \& \alpha \in H_{q}\left(B Z\left(G_{s}, n-1\right)\right)\right\} .
$$

The boundary operator $d_{1}: E_{p, q}^{1} \rightarrow E_{p-1, q}^{1}$ is given by

$$
d_{1}(s \otimes \alpha)=\sum_{i=0}^{p}(-1)^{i} s_{i} \otimes r_{s, s_{i}}(\alpha),
$$

where $s_{i}$ is the $i$ th face of $s$. We should like to show that

$$
E_{0,0}^{2}=\mathbf{Z} \text { and } E_{p, q}^{2}=0 \text { for } 0<p+q<w(G, n)-n .
$$

In order to compute $E_{p, q}^{2}$, we filter $E^{1}$ by

$$
F_{j} E^{1}=\langle s \otimes \alpha|| \bar{s} \mid \leq j\rangle .
$$

Denote the resulting spectral by $\bar{E}$. We remark that this shall not be a first quadrant spectral sequence. The $\bar{E}^{1}$-term decomposes in the form

$$
\bar{E}_{r, t}^{1}=\bigoplus\left\{\bar{E}_{M, t}^{1}|M \subseteq V \&| M \mid=r \& 2^{M} \cap G \neq \varnothing\right\}, \quad r+t=p,
$$

where $\bar{E}_{M, t}^{1}$ is the (appropriately shifted) homology of the complex $C_{M}$ given by

$$
C_{M, p}=\left\{s^{\prime} \otimes \alpha \mid \bar{s}=M \& \operatorname{dim} s=p \& \alpha \in H_{q}\left(B Z\left(G_{M}, n-1\right)\right)\right\} .
$$

Thus,

$$
\bar{E}_{M, t}^{1}=\widetilde{H}_{p-1}\left(B W(G, M), H_{q}\left(B Z\left(G_{M}, n-1\right)\right)\right)
$$

where

$$
W(G, M)=\left\{P \subseteq M \mid P \neq M \& 2^{P} \cap G \neq \varnothing\right\}
$$

(the ordering is by inclusion). Also define

$$
V(G, M)=\left\{P \subseteq M \mid P \neq M \& 2^{P} \cap G=\varnothing\right\} .
$$

Now observe carefully that $B W(\{\varnothing\}, M)$ is homeomorphic (not just equivalent!) to the sphere $S^{r-2}$. Moreover, $B V(G, M)$ is a deformation retract of $B W(\{\varnothing\}, M) \backslash B W(G, M)$. By the Alexander duality theorem,

$$
\bar{E}_{M, t}^{1}=\widetilde{H}^{-t-2}\left(B V(G, M), H_{q}\left(B Z\left(G_{M}, n-1\right)\right)\right) .
$$

Now $B V(G, M)$ has dimension $e(s)-1$ (recall that $s$ is the simplex with $\bar{s}=M)$. Consequently,

$$
\bar{E}_{M, t}^{1}=0 \text { if } e(s)+t+1<0 .
$$

The latter is equivalent to $p-|\bar{s}|+e(s)+1<0, \quad p<|\bar{s}|-e(s)-1$. Also, if $i=p+q<w(G, n)-n$ and $e(s)+t+1 \geq 0$ then $p=|\bar{s}|-e(s)-1$ (the extremal value) and

$$
\begin{aligned}
q & <w(G, n)-n-p=w(G, n)-|\bar{s}|+e(s)-(n-1) \\
& \leq w\left(G_{M}, n-1\right)-(n-1)
\end{aligned}
$$


by (4.1.2). This is exactly the range where $H_{q}\left(B Z\left(G_{M}, n-1\right)\right)$ vanishes by the induction hypothesis, unless, of course, $q=0$. We have thus shown that $\bar{E}_{*, *}^{1}=0$ if $q \neq 0$ and hence

$$
E_{p, q}^{2}=0 \text { for } q \neq 0
$$

in the desired range. For the case $q=0$, we have a different method of computing $E_{p, 0}^{2}$. Let

$$
\begin{aligned}
T=\left\{M \mid\left(\exists P_{1}, \ldots, P_{n} \in G\right) P_{1} \subseteq M \& P_{2}, \ldots, P_{n} \subseteq N \backslash M \&\right. \\
\left.\quad\left(i \neq j \Rightarrow P_{i} \cap P_{j}=\varnothing\right)\right\}
\end{aligned}
$$

(ordering is by inclusion). Then $E_{p, 0}^{2}=H_{p}(B T, \mathbf{Z})$.

We prove the following

4.1.4 Claim. Let $M \subseteq N$ be an (inclusion-) maximal set with $2^{M} \cap G=\varnothing$. Then

(1) $|N \backslash M| \geq w(G, n)$.

(2) For any $M \subseteq W$ such that $1 \leq|W \backslash M| \leq w(G, n)-n+1$ we have $W \in T$.

Proof of Claim. (1) is obvious. To see (2), suppose the contrary. Then let $F_{1}, \ldots, F_{t}, t \leq n-2$, be a maximal system (with respect to $t$ ) of disjoint elements of $2^{N \backslash W} \cap G$. We may also choose all the $F_{i}$ minimal with respect to inclusion. Choose $x_{i} \in F_{i}$. Then the sets $M, F_{i} \backslash\left\{x_{i}\right\}, i=1, \ldots, t$, and $N \backslash\left(W \cup \bigcup_{i=1}^{t} F_{i}\right)$ contain no element of $G$ as a subset. The cardinality of the complement of their union is $\leq w(G, n)-n+1+t \leq w(G, n)-1$, contradicting the definition of $w(G, n)$.

Now let

$$
S=\{M \subseteq N \mid M \neq N \& M \neq \varnothing\}, \quad Q=S \backslash T .
$$

The classifying space $B S$ is homeomorphic to $S^{|N|-2}$ and hence $B Q$ and $B T$ are $(|N|-3)$-dual. In other words,

$$
\left.\widetilde{H}_{p}(B T)=\widetilde{H}^{|N|-3-p}\right)(B Q) .
$$

By Claim 4.1.4,

$$
\operatorname{dim} B Q \leq|N|-3-w(G, n)+n
$$

and hence

$$
\widetilde{H}_{p}(B T)=0 \text { for } p<w(G, n)-n .
$$

This concludes the proof of (4.1.3) and thus of Theorem 4.1.

\section{ACKNOWLEDGMENT}

I am indebted to J. Greenlees, J. P. May, and G. Triantafillou for significant discussions. 


\section{REFERENCES}

1. N. Alon, P. Frankl, and L. Lovász, The chromatic number of Kneser hypergraphs, Trans. Amer. Math. Soc. 298 (1986), 359-370.

2. G. E. Bredon, Equivariant cohomology theories, Lecture Notes in Math., vol. 34, SpringerVerlag, 1967.

3. P. Erdös, Problems and results in combinatorial analysis, Colloq. Internat. Theor. Combin. (Rome 1973), Acad. Naz. Lincei, Rome, 1976, pp. 3-17.

4. M. Kneser, Aufgabe 300, Jber. Deutsch. Math. Verein 58 (1955).

5. L. Lovász, Kneser's conjecture, chromatic number and homotopy, J. Combin. Theory Ser. A 25 (1978), 319-324.

6. J. P. May, Simplicial objects in algebraic topology, Van Nostrand Mathematical Studies, Van Nostrand, 1967.

Department of Mathematics, The University of Chicago, Chicago, Illinois 60637 